\title{
A Computational Method for Volterra Integro-Differential Equation
}

\author{
Erkan ÇİMEN
}

Van Yuzuncu Yil University, Faculty of Education, Department of Mathematics, Van, Turkey

Geliş / Received: 21/06/2018, Kabul / Accepted: 17/10/2018

\begin{abstract}
In this paper, we examine the initial value problem for a linear first order Volterra integro-differential equation. In order to solve the problem computationally, we present a novel finite difference method, which is based on the method of integral identities with the use of the basis functions and interpolating quadrature rules with remainder term in integral form. Furthermore, as a consequence of error analysis the method is proved to be first-order convergent in the discrete maximum norm. Finally, an example is provided to support our theoretical results.
\end{abstract}

Keywords: Error Estimate, Finite Difference Method, Initial Value Problem, Volterra Integro-Differential Equation

\section{Volterra İntegro-Diferansiyel Denklem İçin Bir Çözüm Yöntemi}

$\ddot{O} \mathbf{z}$

$\mathrm{Bu}$ çalışmada, lineer birinci mertebeden Volterra integro-diferansiyel denklem içeren başlangıç değer problemini ele almaktayız. Bu problemi nümerik olarak çözmek için yeni bir sonlu fark metodu veriyoruz. Bu metot, kalan terimi integral biçiminde olan interpolasyon quadratür formülleri ve üstel baz fonksiyonunu içeren integral özdeşliklerinden meydana gelmektedir. Ayrıca, bu metodun hata analizinin bir sonucu olarak, ayrık maksimum normda birinci mertebeden yakınsaklığı ispatlandı. Son olarak, elde edilen teorik sonuçları destekleyen nümerik örnek verildi.

Anahtar Kelimeler: Başlangıç-Değer Problemi, Hata Değerlendirmesi, Sonlu Fark Metodu, Volterra İntegroDiferansiyel Denklem

\section{Introduction}

In this work, we are concerned with the following Volterra integro-differential initial value problem:

$$
\begin{aligned}
\mathrm{Lu}:= & \mathrm{u}^{\prime}(\mathrm{t})+\mathrm{a}(\mathrm{t}) \mathrm{u}(\mathrm{t})=\mathrm{f}(\mathrm{t}) \\
& +\int_{0}^{\mathrm{t}} \mathrm{K}(\mathrm{t}, \mathrm{s}) \mathrm{u}(\mathrm{s}) \mathrm{ds}, \mathrm{t} \in \mathrm{I}, \\
& \mathrm{u}(0)=A
\end{aligned}
$$

where $I=(0, T]$ and $A$ is a given constant. $a(t), f(t)$ and $K(t, s)$ are given sufficiently smooth functions on $\overline{\mathrm{I}}=[0, \mathrm{~T}] \_$and $\overline{\mathrm{I}} \times \overline{\mathrm{I}}$, respectively and moreover

$\mathrm{a}(\mathrm{t}) \geq \alpha>0$.
Volterra integro-differential equations (VIDEs) arise widely in physics, chemistry, biology and engineering applications modelled by initial value problems (Jerri, 1999; Gaetano and Arino, 2000; Song and Baker, 2004). In recent years, it seems that the studies on these problems are being taken more and more into consideration, both in terms of modeling and solution of them. Existence and uniqueness of solution to VIDEs is discussed in books Volterra (1959); Hackbusch (1995); Lakshmikantham and Rao (1995); Jerri (1999); Burton (2005) and the references therein. Furthermore, there are many researchers who have investigated asymptotic expansion and numerical 
approaches for this type equations (Chang, 1982; Hackbusch, 1995; Lakshmikantham and Rao, 1995; Kythe and Puri, 2002; Rahman, 2007; Babolian and Shamloo, 2008; Mehdiyeva et al., 2011; Wazwaz, 2011). The authors in Hoppensteadt et al. (2007); Fazeli and Hojjati (2015); Okayama (2018) are studied the numerical solutions of VIDEs by using various methods, such as collocation method, Runge-Kutta method, Sinc-Nyström method. Nevertheless, authors in Amiraliyev and Sevgin (2006); Amiraliyev and Yilmaz (2014); Kudu et al. (2016) are suggested numerical solutions of VIDEs by using fitted difference method. In this paper, we are present a novel finite-difference scheme on a uniform mesh to approximate (1.1-(1.2). This approach based on the method of integral identities with the use of interpolating quadrature rules with the weight and remainder terms in integral form. As a consequence of the method, in local truncation errors contain only first order derivative of the exact solution and hence eases analysis of the convergence. Our aim of this paper is to approximate for solving (1.1)-(1.2) using a numerical method based on the technique in work of Amiraliyev and Yilmaz (2014). The remainder paper is organized as follows. In Section 2, we put forward a priori estimates of the exact solution of (1.1)-(1.2). In Section 3, we introduce finite difference discretization of the problem (1.1)-(1.2). The error analysis of the approximate solution of the problem is present in Section 4. Furthermore, we prove convergence of our method in the discrete maximum norm. The algorithm for solving the difference problem and the numerical results is formulated in Section 5. Finally, a summary of the main conclusions of the paper are also given.

Notation: Henceforth, $\mathrm{C}$ indicates a generic positive constant. In addition, some particular fixed constants of this type are denoted by subscripting $\mathrm{C}$. We shall use $\|\mathrm{g}\|_{\infty}=$ $\max _{t \in \overline{\mathrm{I}}}|\mathrm{g}(\mathrm{t})|$, for any $\mathrm{g} \in \mathrm{C}(\overline{\mathrm{I}})$.

\section{The Properties of The Exact Solution}

Here, we give some important properties for the solution of our problem, which are needed in later sections for the analysis of appropriate the numerical solution.

Lemma 2.1: Let $a(t), f(t) \in C(\bar{I})$ and $K(t, s) \in$ $\mathrm{C}(\overline{\mathrm{I}} \times \overline{\mathrm{I}})$. Then for the solution $\mathrm{u}$ of the problem (1.1)-(1.2) the following estimates hold:

$$
\begin{aligned}
& \|\mathrm{u}\|_{\infty} \leq \mathrm{C}_{0}, \\
& \left\|\mathrm{u}^{\prime}\right\|_{\infty} \leq \mathrm{C}
\end{aligned}
$$

where

$$
\begin{aligned}
& \mathrm{C}_{0}=\left(|\mathrm{A}|+\alpha^{-1}\|\mathrm{f}\|_{\infty}\right) \mathrm{e}^{\alpha^{-1} \overline{\mathrm{K}} \mathrm{T}}, \\
& \overline{\mathrm{K}}=\max _{\overline{\mathrm{I}} \times \overline{\mathrm{I}}}|\mathrm{K}(\mathrm{t}, \mathrm{s})| .
\end{aligned}
$$

Proof: From (1.1), we have

$$
\begin{gathered}
u(t)=u(0) e^{-\int_{0}^{t} a(\eta) d \eta} \\
+\int_{0}^{t}\left[f(s)+\int_{0}^{s} K(s, \xi) u(\xi) d \xi\right] e^{-\int_{s}^{t} a(\eta) d \eta} d s, \\
|u(t)| \leq|u(0)| e^{-\alpha t}+ \\
\int_{0}^{t}\left[|f(s)|+\int_{0}^{s}|K(s, \xi)||u(\xi)| d \xi\right] e^{-\alpha(t-s)} d s \\
\leq|A| e^{-\alpha t}+\alpha^{-1}\|f\|_{\infty}\left(1-e^{-\alpha t}\right) \\
+\alpha^{-1} \bar{K}\left(1-e^{-\alpha t}\right) \int_{0}^{t}|u(\xi)| d \xi \\
\leq|A|+\alpha^{-1}\|f\|_{\infty}+\alpha^{-1} \bar{K} \int_{0}^{t}|u(\xi)| d \xi .
\end{gathered}
$$

Now, applying the Gronwall's inequality to this inequality, we get

$$
|\mathrm{u}(\mathrm{t})| \leq\left(|\mathrm{A}|+\alpha^{-1}\|\mathrm{f}\|_{\infty}\right) \mathrm{e}^{\alpha^{-1} \overline{\mathrm{K}} \mathrm{t}}
$$

which leads to (2.1). Also, from (1.1) we have

$$
\begin{aligned}
& \left|\mathrm{u}^{\prime}(\mathrm{t})\right| \leq|\mathrm{a}(\mathrm{t})||\mathrm{u}(\mathrm{t})|+|\mathrm{f}(\mathrm{t})| \\
& +\int_{0}^{\mathrm{t}}|\mathrm{K}(\mathrm{t}, \mathrm{s})||\mathrm{u}(\mathrm{s})| \mathrm{d} \mathrm{s} \\
& \leq\|\mathrm{a}\|_{\infty} \mathrm{C}_{0}+\|\mathrm{f}\|_{\infty}+\overline{\mathrm{K}} \mathrm{C}_{0} \int_{0}^{\mathrm{t}} \mathrm{ds} \\
& \leq\|\mathrm{f}\|_{\infty}+\mathrm{C}_{0}\left(\|\mathrm{a}\|_{\infty}+\overline{\mathrm{K}} \mathrm{T}\right)
\end{aligned}
$$

which immediately leads to (2.2)

\section{The Difference Scheme}

We introduce the uniform mesh on the I:

$$
\begin{gathered}
\omega_{N}=\left\{t_{i}=i \tau, i=1,2, \ldots, N ; \tau=T / N\right\}, \\
\bar{\omega}_{N}=\omega_{N} \cup\{0\} .
\end{gathered}
$$

In order to simplify the notation, we define $g_{i}=$ $\mathrm{g}\left(\mathrm{t}_{\mathrm{i}}\right)$ for any function $\mathrm{g}(\mathrm{t})$ and $\mathrm{y}_{\mathrm{i}}$ represents an approximation of $u(t)$ at $t_{i}$ also $g_{i-\frac{1}{2}}=g\left(t_{i}-\frac{\tau}{2}\right)$. Also, for any mesh function $g_{i}$ defined on $\omega_{N}$ we use 


$$
\begin{aligned}
& g_{\bar{t}, i}=\frac{g_{i}-g_{i-1}}{\tau}, \\
& \|g\|_{\infty, \omega_{N}}=\max _{1 \leq i \leq N}\left|g_{i}\right| .
\end{aligned}
$$

We give the difference approximation of Eq. (1.1), by using the following identity:

$$
\begin{aligned}
& \tau^{-1} \int_{t_{i-1}}^{t_{i}} \operatorname{Lu}(\mathrm{t}) \varphi_{\mathrm{i}}(\mathrm{t}) \mathrm{dt}=\tau^{-1} \int_{\mathrm{t}_{\mathrm{i}-1}}^{\mathrm{t}_{\mathrm{i}}}[\mathrm{f}(\mathrm{t}) \\
& \left.\quad+\int_{0}^{\mathrm{t}} \mathrm{K}(\mathrm{t}, \mathrm{s}) \mathrm{u}(\mathrm{s}) \mathrm{ds}\right] \varphi_{\mathrm{i}}(\mathrm{t}) \mathrm{dt}, 1 \leq \mathrm{i} \leq \mathrm{N}
\end{aligned}
$$

with the basis functions

$$
\varphi_{\mathrm{i}}(\mathrm{t})=\mathrm{e}^{-\int_{\mathrm{t}}^{\mathrm{t}_{\mathrm{i}}} \mathrm{a}(\mathrm{s}) \mathrm{ds}}, \mathrm{t}_{\mathrm{i}-1} \leq \mathrm{t} \leq \mathrm{t}_{\mathrm{i}}
$$

which is the solution of the problem

$$
\left\{\begin{array}{l}
-\varphi_{\mathrm{i}}{ }^{\prime}(\mathrm{t})+\mathrm{a}(\mathrm{t}) \varphi_{\mathrm{i}}(\mathrm{t})=0, \mathrm{t}_{\mathrm{i}-1}<\mathrm{t} \leq \mathrm{t}_{\mathrm{i}} \\
\varphi_{\mathrm{i}}\left(\mathrm{t}_{\mathrm{i}}\right)=1 .
\end{array}\right.
$$

The relation (3.1) is rewritten by taking into account the equation (1.1), we get

$$
\begin{aligned}
\tau^{-1} \int_{t_{i-1}}^{t_{i}} u^{\prime}(t) \varphi_{i}(t) d t \\
\quad+\tau^{-1} \int_{t_{i-1}}^{t_{i}} a(t) u(t) \varphi_{i}(t) d t \\
=\tau^{-1} \int_{t_{i-1}}^{t_{i}} f(t) \varphi_{i}(t) d t \\
\quad+\tau^{-1} \int_{t_{i-1}}^{t_{i}}\left[\int_{0}^{t} K(t, s) u(s) d s\right] \varphi_{i}(t) d t .
\end{aligned}
$$

Next, using formulas (2.1) and (2.2) from Amiraliyev and Mamedov (1995) on each interval $\left(t_{i-1}, t_{i}\right)$ left hand side (3.3) and taking into account (3.2) we have following precise relation

$$
\begin{aligned}
& \tau^{-1} \int_{t_{i-1}}^{t_{i}} u^{\prime}(t) \varphi_{i}(t) d t \\
& +\tau^{-1} \int_{t_{i-1}}^{t_{i}} a(t) u(t) \varphi_{i}(t) d t=A_{i} u_{\bar{t}, i}+B_{i} u_{i} .
\end{aligned}
$$

where

$$
\begin{aligned}
A_{i}= & \tau^{-1} \int_{t_{i-1}}^{t_{i}} \varphi_{i}(t) d t \\
& \quad+\tau^{-1} \int_{t_{i-1}}^{t_{i}}\left(t-t_{i}\right) a(t) \varphi_{i}(t) d t \\
B_{i}= & \tau^{-1} \int_{t_{i-1}}^{t_{i}} a(t) \varphi_{i}(t) d t .
\end{aligned}
$$

For the integral term from (3.3), after applying the appropriate quadrature rules, we have

$$
\tau^{-1} \int_{t_{i-1}}^{t_{i}}\left[\int_{0}^{t} K(t, s) u(s) d s\right] \varphi_{i}(t) d t
$$

$$
\begin{aligned}
= & \tau^{-1} \int_{t_{i-1}}^{t_{i}} \varphi_{i}(t) d t \int_{0}^{t_{i}-\frac{1}{2}} K\left(t_{i-\frac{1}{2}}, s\right) u(s) d s \\
& +R_{i}^{(1)} \\
= & {\left[\tau^{-1} \int_{t_{i-1}}^{t_{i}} \varphi_{i}(t) d t\right] \tau \sum_{j=0}^{i-1} K\left(t_{i-\frac{1}{2}}, s_{j}\right) u_{j} } \\
& +R_{i}^{(1)}+R_{i}^{(2)}
\end{aligned}
$$

where

$$
\begin{aligned}
& \mathrm{R}_{\mathrm{i}}^{(1)}= \\
& \tau^{-1} \int_{t_{i-1}}^{t_{i}} d t \varphi_{i}(t) \int_{t_{i-1}}^{t_{i}} \frac{\partial}{\partial t}\left[\int_{0}^{t} K(t, s) u(s) d s\right] \\
& \times\left[\mathrm{T}_{0}(\mathrm{~s}-\mathrm{t})-\mathrm{T}_{0}\left(\mathrm{t}_{\mathrm{i}-\frac{1}{2}}-\mathrm{t}\right)\right] \mathrm{dt} \quad(3.5) \\
& \mathrm{R}_{\mathrm{i}}^{(2)}=\sum_{\mathrm{j}=1}^{\mathrm{i}-1} \int_{\mathrm{t}_{\mathrm{j}-\frac{1}{2}}}^{\mathrm{t}+\frac{1}{2}}\left[\mathrm{t}_{\mathrm{j}+\frac{1}{2}}-\mathrm{t}-\tau \mathrm{T}_{0}\left(\mathrm{t}_{\mathrm{j}}-\mathrm{t}\right)\right] \times \\
& \times \frac{d}{d t}\left[K\left(t_{i-\frac{1}{2}}, t\right) u(t)\right] d t
\end{aligned}
$$

and

$$
\mathrm{T}_{0}(\lambda)=1, \lambda>0 ; \mathrm{T}_{0}(\lambda)=0, \lambda \leq 0 .
$$
$u\left(t_{i}\right)$ :

Hereby, we write the exact relation for

$$
\begin{aligned}
\ell u_{i}:=A_{i} u_{\bar{t}, i} & +B_{i} u_{i}=F_{i} \\
& +\tau C_{i} \sum_{j=0}^{i-1} K\left(t_{i-\frac{1}{2}}, t_{j}\right) u_{j}+R_{i}
\end{aligned}
$$

with

$$
\begin{aligned}
& \mathrm{C}_{\mathrm{i}}=\tau^{-1} \int_{\mathrm{t}_{\mathrm{i}-1}}^{\mathrm{t}_{\mathrm{i}}} \varphi_{\mathrm{i}}(\mathrm{t}) \mathrm{dt}, \\
& \mathrm{F}_{\mathrm{i}}=\tau^{-1} \int_{\mathrm{t}_{\mathrm{i}-1}}^{\mathrm{t}_{\mathrm{i}}} \mathrm{f}(\mathrm{t}) \varphi_{\mathrm{i}}(\mathrm{t}) \mathrm{dt}, \\
& \mathrm{R}_{\mathrm{i}}=\mathrm{R}_{\mathrm{i}}^{(1)}+\mathrm{R}_{\mathrm{i}}^{(2)}
\end{aligned}
$$

where $A_{i}, B_{i}$ and $R_{i}^{(k)}(k=1,2)$ are determined by (3.4), (3.5), (3.6), respectively.

By virtue of (3.7) we suggest the following difference scheme for approximating (1.1)-(1.2):

$$
\begin{aligned}
\ell \mathrm{y}_{\mathrm{i}} & :=\mathrm{A}_{\mathrm{i}} \mathrm{y}_{\mathrm{t}, \mathrm{i}}+\mathrm{B}_{\mathrm{i}} \mathrm{y}_{\mathrm{i}}=\mathrm{F}_{\mathrm{i}} \\
& +\tau \mathrm{C}_{\mathrm{i}} \sum_{\mathrm{j}=0}^{\mathrm{i}-1} \mathrm{~K}\left(\mathrm{t}_{\mathrm{i}-\frac{1}{2}}, \mathrm{t}_{\mathrm{j}}\right) \mathrm{y}_{\mathrm{j}}, \\
\mathrm{y}_{0} & =\mathrm{A} .
\end{aligned}
$$

\section{Stability Bound and Convergence}

In order to examine the convergence of the method, the error function $z_{i}=y_{i}-u_{i},(1 \leq$ $\mathrm{i} \leq \mathrm{N}$ ) which is the solution of the following discrete problem

$$
\begin{gathered}
\ell \mathrm{z}_{\mathrm{i}}=\mathrm{R}_{\mathrm{i}}, 1 \leq \mathrm{i} \leq \mathrm{N}, \\
\mathrm{z}_{0}=0 .
\end{gathered}
$$


Lemma 4.1: Let $\left|G_{i}\right| \leq \bar{G}_{i}$ and $\bar{G}_{i}$ be a nondecreasing function.

$$
\begin{aligned}
& \ell \mathrm{v}_{\mathrm{i}}:=\mathrm{A}_{\mathrm{i}} \mathrm{v}_{\mathrm{t}, \mathrm{i}}+\mathrm{B}_{\mathrm{i}} \mathrm{v}_{\mathrm{i}}=\mathrm{G}_{\mathrm{i}}, 1 \leq \mathrm{i} \leq \mathrm{N} \\
& \mathrm{v}_{0}=\mathrm{A} .
\end{aligned}
$$

Then the solution of difference problem (4.3)-(4.4) holds:

$$
\left|\mathrm{v}_{\mathrm{i}}\right| \leq|\mathrm{A}|+\alpha^{-1} \overline{\mathrm{G}}_{\mathrm{i}}, 1 \leq \mathrm{i} \leq \mathrm{N} .
$$

Proof: The proof is almost identical that of (Amiraliyev and Yilmaz, 2014).

Lemma 4.2: Let $K(t, s) \in C^{1}(\bar{I} \times \bar{I})$. Then for the truncation error $\mathrm{R}_{\mathrm{i}}$, the following estimate holds

$$
\|\mathrm{R}\|_{\infty, \omega_{\mathrm{N}}} \leq \mathrm{CN}^{-1} \text {. }
$$

Proof: Since $0<\varphi_{\mathrm{i}}(\mathrm{t}) \leq 1$

$$
\left|\mathrm{R}_{\mathrm{i}}^{(1)}\right| \leq \int_{\mathrm{t}_{\mathrm{i}-1}}^{\mathrm{t}_{\mathrm{i}}}\left|\frac{\partial}{\partial \mathrm{t}}\left[\int_{0}^{\mathrm{t}} \mathrm{K}(\mathrm{t}, \mathrm{s}) \mathrm{u}(\mathrm{s}) \mathrm{ds}\right]\right| \mathrm{dt}
$$

and after applying Leibniz rule, we get

$$
\begin{aligned}
\left|\mathrm{R}_{\mathrm{i}}^{(1)}\right| \leq & \int_{\mathrm{t}_{\mathrm{i}-1}}^{\mathrm{t}_{\mathrm{i}}}|\mathrm{K}(\mathrm{t}, \mathrm{t}) \mathrm{u}(\mathrm{t})| \mathrm{dt} \\
& +\int_{\mathrm{t}_{\mathrm{i}-1}}^{\mathrm{t}_{\mathrm{i}}}\left|\left[\int_{0}^{\mathrm{t}} \frac{\partial}{\partial \mathrm{t}} \mathrm{K}(\mathrm{t}, \mathrm{s}) \mathrm{u}(\mathrm{s}) \mathrm{ds}\right]\right| \mathrm{dt} .
\end{aligned}
$$

Hence the estimate $\left|R_{i}^{(1)}\right| \leq C \tau$ is easily obtained.

Next, from (3.6)

$$
\begin{gathered}
\left|R_{i}^{(2)}\right| \leq 2 \tau \sum_{j=1}^{i-1} \int_{t_{j-\frac{1}{2}}}^{t} t_{j+\frac{1}{2}}\left[\frac{d}{d t} K\left(t_{i-\frac{1}{2}}, t\right) u(t)\right. \\
\left.+K\left(t_{i-\frac{1}{2}}, t\right) u^{\prime}(t)\right] d t
\end{gathered}
$$

taking into account Lemma 2.1, we have

$$
\begin{aligned}
\left|\mathrm{R}_{\mathrm{i}}^{(2)}\right| & \leq 2 \tau^{2}(\mathrm{~N}-1)\left(\mathrm{C}_{0}\left|\frac{\partial}{\partial \mathrm{s}} \mathrm{K}(\mathrm{t}, \mathrm{s})\right|+\mathrm{C} \overline{\mathrm{K}}\right) \\
& \leq \mathrm{C} \tau .
\end{aligned}
$$

Lemma 4.3: The solution of (4.1)-(4.2) satisfies the following estimate

$$
\|\mathrm{z}\|_{\infty, \omega_{\mathrm{N}}} \leq \mathrm{C}\|\mathrm{R}\|_{\infty, \omega_{\mathrm{N}}} .
$$

Proof: From the solution of (4.1)-(4.2):

$$
\ell \mathrm{z}_{\mathrm{i}}:=A_{\mathrm{i}} \mathrm{z}_{\overline{\mathrm{t}} \mathrm{i}}+\mathrm{B}_{\mathrm{i}} \mathrm{z}_{\mathrm{i}}
$$

$$
=\tau C_{i} \sum_{j=0}^{i-1} K\left(t_{i-\frac{1}{2}}, t_{j}\right) z_{j}+R_{i}
$$

and taking account Lemma 4.1

$$
\left|\mathrm{z}_{\mathrm{i}}\right| \leq \alpha^{-1} \tau \sum_{\mathrm{j}=0}^{\mathrm{i}-1} \overline{\mathrm{K}}\left|\mathrm{z}_{\mathrm{j}}\right|+\alpha^{-1}\|\mathrm{R}\|_{\infty} .
$$

From here by using the difference analogue of Gronwall's inequality, we get

$$
\left|\mathrm{z}_{\mathrm{i}}\right| \leq \alpha^{-1}\|\mathrm{R}\|_{\infty} \mathrm{e}^{\alpha^{-1} \overline{\mathrm{K}} \mathrm{t}_{\mathrm{i}}}
$$

and this immediately leads to (4.5).

Finally, we give the main convergence result.

Theorem 4.1: Let $\mathrm{u}$ be the solution of (1.1)(1.2) and y the solution (3.8)-(3.9). Then

$$
\|\mathrm{y}-\mathrm{u}\|_{\infty, \bar{\omega}_{\mathrm{N}}} \leq \mathrm{CN}^{-1} \text {. }
$$

Proof: By combining the previous Lemmas 4.2 and 4.3 , we can immediately prove.

\section{Algorithm and Numerical Results}

In this section, we present numerical results obtained by applying the numerical method (3.8)-(3.9) to the particular problem. We rewritten difference scheme (3.8)

$$
\begin{aligned}
y_{i}= & \frac{A_{i}}{A_{i}+\tau B_{i}} y_{i-1}+\frac{\tau F_{i}}{A_{i}+\tau B_{i}} \\
& +\frac{\tau^{2} C_{i}}{A_{i}+\tau B_{i}} \sum_{j=0}^{i-1} K\left(t_{i-\frac{1}{2}}, t_{j}\right) y_{j}
\end{aligned}
$$

from hence, for $1 \leq \mathrm{i} \leq \mathrm{N}$ with together $\mathrm{y}_{0}=\mathrm{A}$, we get any $y_{i}$.

Example 5.1 Now, we consider the test problem

$$
\begin{aligned}
\mathrm{u}^{\prime}(\mathrm{t}) & +2 \mathrm{u}(\mathrm{t})=1-\mathrm{t} \\
& +\int_{0}^{\mathrm{t}}(\mathrm{t}-\mathrm{s}) \mathrm{u}(\mathrm{s}) \mathrm{ds}, \mathrm{t} \in(0,1], \\
\mathrm{u}(0)= & 1 .
\end{aligned}
$$

The exact solution is given by

$$
\mathrm{u}(\mathrm{t})=\mathrm{e}^{-\mathrm{t}} \text {. }
$$

We define the exact error $E_{i}^{N}$, the computed maximum pointwise error $\mathrm{E}^{\mathrm{N}}$ as follows, respectively:

$$
E_{i}^{N}=\left|y_{i}-u_{i}\right|, E^{N}=\max _{0 \leq i \leq N} E_{i}^{N}
$$

where $\mathrm{y}$ is the numerical approximation to $\mathrm{u}$ for various values of N. For Example 5.1 the computational results obtained by present method are given in the Tables 1-3. 
Table 1. Computational results for $N=64$

\begin{tabular}{llll}
\hline $\mathrm{t}_{\mathrm{i}}$ & $\mathrm{u}\left(\mathrm{t}_{\mathrm{i}}\right)$ & $\mathrm{y}\left(\mathrm{t}_{\mathrm{i}}\right)$ & $\mathrm{E}_{\mathrm{i}}^{\mathrm{N}}$ \\
\hline 0.000 & 1.0 & 1.0 & 0.0 \\
0.125 & 0.8824969 & 0.8825552 & $5.83341 \mathrm{E}-5$ \\
0.250 & 0.7788008 & 0.7790124 & $2.11611 \mathrm{E}-4$ \\
0.375 & 0.6872893 & 0.6877284 & $4.39125 \mathrm{E}-4$ \\
0.500 & 0.6065307 & 0.6072557 & $7.25078 \mathrm{E}-4$ \\
0.625 & 0.5352614 & 0.5363190 & $1.05760 \mathrm{E}-3$ \\
0.750 & 0.4723666 & 0.4737945 & $1.42799 \mathrm{E}-3$ \\
0.875 & 0.4168620 & 0.4186921 & $1.83006 \mathrm{E}-3$ \\
1.000 & 0.3678794 & 0.3701391 & $2.25968 \mathrm{E}-3$ \\
\hline
\end{tabular}

Table 2. Computational results for $\mathrm{N}=128$

\begin{tabular}{llll}
\hline $\mathrm{t}_{\mathrm{i}}$ & $\mathrm{u}\left(\mathrm{t}_{\mathrm{i}}\right)$ & $\mathrm{y}\left(\mathrm{t}_{\mathrm{i}}\right)$ & $\mathrm{E}_{\mathrm{i}}^{\mathrm{N}}$ \\
\hline 0.000 & 1.0 & 1.0 & 0.0 \\
0.125 & 0.8824969 & 0.8825256 & $2.86513 \mathrm{E}-5$ \\
0.250 & 0.7788008 & 0.7789057 & $1.04941 \mathrm{E}-4$ \\
0.375 & 0.6872893 & 0.6875077 & $2.18464 \mathrm{E}-4$ \\
0.500 & 0.6065307 & 0.6068919 & $3.61281 \mathrm{E}-4$ \\
0.625 & 0.5352615 & 0.5357889 & $5.27430 \mathrm{E}-4$ \\
0.750 & 0.4723666 & 0.4730791 & $7.12533 \mathrm{E}-4$ \\
0.875 & 0.4168620 & 0.4177755 & $9.13484 \mathrm{E}-4$ \\
1.000 & 0.3678794 & 0.3690076 & $1.12820 \mathrm{E}-3$ \\
\hline
\end{tabular}

Table 3. Maximum pointwise errors for Example 5.1

\begin{tabular}{cccc}
\hline $\mathrm{N}$ & $\mathrm{E}^{\mathrm{N}}$ & $\mathrm{N}$ & $\mathrm{E}^{\mathrm{N}}$ \\
\hline 32 & $4.53203 \mathrm{E}-3$ & 256 & $5.63682 \mathrm{E}-4$ \\
64 & $2.25968 \mathrm{E}-3$ & 512 & $2.81736 \mathrm{E}-4$
\end{tabular}

$128 \quad 1.12820 \mathrm{E}-3 \quad 1024 \quad 1.40842 \mathrm{E}-4$

\section{Conclusions}

In this work, we have developed a novel method for approximating solution of the initial-value problem for a linear first order Volterra integro-differential equation. The method was based on an exponentially difference scheme on a uniform mesh. As results from the method, first order convergence in the discrete maximum norm was obtained. However, using the method, we were solved a numerical and the obtained results were displayed in Tables 1-3. These results were achieved to show the efficiency and accuracy of the our method. Theoretical results represented undergoing research more complicated Volterra integro-differential equations, such as delay Volterra integrodifferential equation.

\section{References}

Amiraliyev, G.M., Mamedov, Y.D. 1995. Difference schemes on the uniform mesh for singularly perturbed pseudoparabolic equations. Turkish Journal of Mathematics, 19, 207-222. 
Amiraliyev, G.M., Sevgin, S. 2006. Uniform difference method for singularly perturbed Volterra integro-differential equations. Applied Mathematics and Computation, 179(2), 731-741.

Amiraliyev, G.M., Yilmaz, B. 2014. Fitted difference method for a singularly perturbed initial value problem. International Journal of Mathematics and Computation, 22(1), 1-10.

Babolian, E., Shamloo, A.S. 2008. Numerical solution of Volterra integral and integro-differential equations of convolution type by using operational matrices of piecewise constant orthogonal functions. Journal of Computational and Applied Mathematics, 214(2), 495-508.

Burton, T.A. 2005. Volterra Integral and Differential Equations 2nd ed, Elsevier, Amsterdam.

Chang, S.H. 1982. On certain extrapolation methods for the numerical solution of integro-differential equations. Mathematics of Computation, 39(159), 165-171.

De Gaetano, A., Arino, O. 2000. Mathematical modelling of the intravenous glucose tolerance test. Journal of Mathematical Biology, 40(2), 136-168.

Fazeli, S., Hojjati, G. 2015. Numerical solution of Volterra integro-differential equations by superimplicit multistep collocation methods. Numerical Algorithms, 68(4), 741-768.

Hackbusch, W. 1995. Integral Equations Theory and Numerical Treatment, Birkhauser Verlag, Basel.

Hoppensteadt, F.C., Jackiewicz, Z., Kowal, B.Z. 2007. Numerical solution of Volterra integral and integrodifferential equations with rapidly vanishing convolution kernels. BIT Numerical Mathematics, 47(2), 325350 .

Jerri, A. 1999. Introduction to Integral Equations with Applications, Wiley, New York.

Khater, A.H., Shamardan A.B., Callebaut D.K., Sakran, M.R.A. 2007. Numerical solutions of integral and integrodifferential equations using Legendre polynomials. Numerical Algorithms, 46(3), 195-218.

Kudu, M., Amirali, I., Amiraliyev, G.M. 2016. A finite-difference method for a singularly perturbed delay integrodifferential equation. Journal of Computational and Applied Mathematics, 308, 379-390.

Kythe, P.K., Puri, P. 2002. Computational Methods for Linear Integral Equations, Springer, New York.

Lakshmikantham, V., Rao, M.R.M. 1995. Theory of Integro-Differential Equations, Gordon and Breach Science Publishers, Amsterdam.

Mehdiyeva, G., Imanova, M., Ibrahimov, V. 2011. Application of the hybrid methods to solving Volterra integrodifferential equations. World Academy of Science, Engineering and Technology International Journal of Mathematical and Computational Sciences, 5(5), 788-792.

Okayama, T. 2018. Theoretical analysis of a Sinc-Nyström method for Volterra integro-differential equations and its improvement. Applied Mathematics and Computation, 324, 1-15.

Rahman, M. 2007. Integral Equations and their Applications, WIT Press, Boston.

Song, Y., Baker, C.T.H. 2004. Qualitative behaviour of numerical approximations to Volterra integro-differential equations, Journal of Computational and Applied Mathematics, 172(1), 101115.

Volterra, V. 1959. Theory of Functionals and of Integral and Integro-differential Equations, Dover Publications, New York, 138-146.

Wazwaz, A.M. 2011. Linear and Nonlinear Integral Equations Methods and Applications, Springer-Verlag, Berlin. 